\title{
LIBERAÇÃO DE NUTRIENTES PELA PALHADA DE MILHETO EM DIFERENTES ESTÁDIOS FENOLÓGICOS ${ }^{(1)}$
}

\author{
Lília Karla Carpim ${ }^{(2)}$, Renato Lara de Assis ${ }^{(3)}$, Antonio Joaquim Braga Pereira Braz ${ }^{(3)}$, \\ Gilson Pereira Silva ${ }^{(3)}$, Fábio Ribeiro Pires ${ }^{(4)}$, Vinícius Cruvinel Pereira ${ }^{(5)}$, Graciely \\ Vilela Gomes ${ }^{(5)} \&$ Alessandro Guerra da Silva ${ }^{(3)}$
}

\begin{abstract}
RESUMO
Este estudo foi realizado na área experimental da Faculdade de Agronomia da FESURV - Universidade de Rio Verde, no sudoeste de Goiás, em um Latossolo Vermelho distroférrico textura argilosa, no período de outubro de 2004 a maio de 2005. Seu objetivo foi avaliar a liberação de nutrientes na palhada de milheto (Pennisetum glaucum), cultivar ADR300, em diferentes estádios fenológicos (préemborrachamento, pré-florescimento e início do florescimento). Utilizou-se essa cultura como cobertura de solo para o sistema plantio direto, em parcelas com área total de $9 \mathrm{~m}^{2}$. O delineamento experimental utilizado foi o de blocos casualizados, com parcelas subdivididas no tempo, com quatro repetições. Para avaliar a liberação de nutrientes, foi utilizado o método de bolsas de decomposição. Após o manejo do milheto, as avaliações de decomposição da palhada foram realizadas em intervalos de 30 dias, até 150 dias após o manejo. $O$ maior acúmulo de nutrientes foi no estádio fenológico de início do florescimento. A dinâmica da liberação de nutrientes foi diferente em todos os estádios fenológicos com exceção para o K; este foi o nutriente mais facilmente liberado, independentemente do estádio fenológico.
\end{abstract}

Termos de indexação: planta de cobertura, bolsas de decomposição, manejo.

(1) Trabalho apresentado no XXXI Congresso Brasileiro de Ciência do Solo, 2007 (Gramado, RS).

(2) Engenheiro Agrônomo, CEP 75900-000 Rio Verde (GO). E-mail: liliacarpim@hotmail.com

(3) Professor da Faculdade de Agronomia, Fesurv - Universidade de Rio Verde. Caixa Postal 104, CEP 75901-970 Rio Verde (GO), E-mail: assis@fesurv.br; braga@fesurv.br; gilson@fesurv.br; silvaag@fesurv.br

(4) Professor do Departamento de Ciências da Saúde, Biológicas e Agrárias, Universidade Federal do Espírito Santo - CEUNES/ UFES. R. Humberto de Almeida Franklin 257, B. Universitário, CEP 29933-415 São Mateus (ES). E-mail: fabiopires@ceunes.ufes.br

(5) Mestrando do Programa de Pós-graduação em Produção Vegetal da Fesurv - Universidade de Rio Verde. E-mail: viniciuscruvinel@yahoo.com.br; gracielyv@hotmail.com 


\title{
SUMMARY: NUTRIENT RELEASE FROM PEARL MILLET IN DIFFERENT PHENOLOGICAL STAGES
}

\begin{abstract}
This study was carried out in an experimental area of the Faculdade de Agronomia of the Universidade de Rio Verde, from October 2004 to May 2005, in a clayey dystroferric Red Latosol (Oxisol). The purpose of the experiment was to evaluate nutrient release from Pennisetum glaucum straw, cv. ADR300, in three different phenological stages (pre-booting, pre-flowering and early flowering). Pennisetum glaucum was used as cover crop in a no tillage system, in a randomized block design, and split plots in time, with four replications, totalizing $9 \mathrm{~m} 2$. To evaluate nutrient release, a decomposition bag system was used. After Pennisetum glaucum management, decomposition was evaluated during 150 days, at intervals of 30 days. Nutrient accumulation was highest at the early flowering stage. Nutrient release dynamics differed in all phenological stages, with exception of $K$, which was the nutrient of easiest release, regardless of the phenological stage.
\end{abstract}

Index terms: cover crop, decomposition bags, management.

\section{INTRODUÇÃO}

A eficácia do sistema plantio direto está relacionada, dentre outros fatores, com a quantidade e qualidade dos resíduos culturais deixados na superfície do solo. A rápida expansão do cultivo de milheto no Brasil, principalmente na região do Cerrado, é atribuída à sua fácil instalação e adaptação às condições desfavoráveis de cultivo, destacando-se: tolerância à seca, crescimento rápido e maior capacidade de ciclagem de nutrientes; alta produção de biomassa; boa adaptação a diferentes níveis de fertilidade; sistema radicular profundo e abundante; facilidade de mecanização; resistência a pragas e doenças; facilidade de produção de semente e aproveitamento para pecuária, decorrente da boa qualidade e da elevada produção de forragem (Teixeira et al., 2005).

$\mathrm{O}$ milheto normalmente é cultivado até à fase de emborrachamento (pré-floração), quando é dessecado e efetuada a semeadura da cultura subseqüente. Entretanto, o manejo do milheto em outros estádios fenológicos, antecedendo a cultura de verão, ainda é pouco estudado. A época de semeadura do milheto é ampla, em razão de sua rusticidade, podendo o cultivo ser realizado de agosto a maio, dependendo da finalidade de uso.

Em estudos para avaliação da composição química da matéria seca da parte aérea do milheto comum, Moraes (2001), Oliveira et al. (2002) e Teixeira et al. (2005) obtiveram valores de matéria seca de $9,65 \mathrm{Mg} \mathrm{ha}^{-1}$ no estádio de pleno florescimento; $14,18 \mathrm{Mg} \mathrm{ha}^{-1} 100$ dias após a semeadura e 2,91 $\mathrm{Mg} \mathrm{ha}^{-1}$ 119 dias após a semeadura, respectivamente, e a quantidade de nutrientes variando para o N ( 49 a 64 $\left.\mathrm{kg} \mathrm{ha}^{-1}\right), \mathrm{P}\left(7\right.$ a $\left.25 \mathrm{~kg} \mathrm{ha}^{-1}\right), \mathrm{K}$ (21 a $\left.166 \mathrm{~kg} \mathrm{ha}^{-1}\right)$, Ca (11 a $\left.106 \mathrm{~kg} \mathrm{ha}^{-1}\right), \mathrm{Mg}\left(5\right.$ a $\left.54 \mathrm{~kg} \mathrm{ha}^{-1}\right)$ e S (4 a $\left.26 \mathrm{~kg} \mathrm{ha}^{-1}\right)$. Moraes (2001) observou após 168 dias de avaliação uma mineralização dos macronutrientes de 96,$8 ; 93,6$; 95,$8 ; 96,9 ; 90,0$ e $82,5 \%$, respectivamente, para $\mathrm{N}$, $\mathrm{P}, \mathrm{K}, \mathrm{Ca}, \mathrm{Mg}$ e S.
Em condições de safrinha no sudoeste de Goiás, Boer et al. (2007), em estudo com milheto cultivar ADR500, no estádio fenológico de pleno florescimento, aos 51 dias da semeadura, obtiveram produção de biomassa seca de $10,80 \mathrm{Mg} \mathrm{ha}^{-1}$ e relação $\mathrm{C} / \mathrm{N}$ de 34 , além de encontrar maior acúmulo de $\mathrm{N}(121,75 \mathrm{~kg}$ $\left.\mathrm{ha}^{-1}\right)$ e K $\left(416,94 \mathrm{~kg} \mathrm{ha}^{-1}\right)$ em relação aos estudos com o milheto comum, pelo fato de o cultivar ADR500 apresentar grande capacidade de produção de matéria seca, ciclagem e acúmulo de nutrientes.

Neste contexto, este trabalho teve como objetivo avaliar a liberação de nutrientes na palhada do milheto, cultivar ADR300, em diferentes estádios fenológicos, antecedendo a semeadura da cultura de verão.

\section{MATERIAL E MÉTODOS}

O experimento foi realizado na área experimental da Faculdade de Agronomia da FESURV Universidade de Rio Verde, no município de Rio Verde, GO, localizado na latitude de $17^{\circ} 47^{\prime} 30^{\prime \prime} \mathrm{S}$, longitude $50^{\circ} 57^{\prime} 44^{\prime \prime} \mathrm{W}$ e altitude de $770 \mathrm{~m}$.

A espécie utilizada no experimento foi o milheto, (Pennisetum glaucum), cultivar ADR300, e o solo da área experimental foi classificado como Latossolo Vermelho distroférrico relevo suave ondulado $\left(0-4 \mathrm{~cm} \mathrm{~m}^{-1}\right)$ textura argilosa. A análise química e física seguiu métodos da Embrapa (1997), com as seguintes características na camada arável $(0-20 \mathrm{~cm})$ por ocasião da instalação do experimento: $\mathrm{pH}$ em $\mathrm{CaCl}_{2}$ de 4,7; $1,34 \mathrm{cmol}_{\mathrm{c}} \mathrm{dm}^{-3} \mathrm{de} \mathrm{Ca} ; 0,85 \mathrm{cmol}_{\mathrm{c}} \mathrm{dm}^{-3} \mathrm{de} \mathrm{Mg} ; 0,82$ $\mathrm{cmol}_{\mathrm{c}} \mathrm{dm}^{-3}$ de K; $4,40 \mathrm{cmol}_{\mathrm{c}} \mathrm{dm}^{-3} \mathrm{de} \mathrm{H}+\mathrm{Al} ; 8,91 \mathrm{mg}$ $\mathrm{dm}^{-3}$ de $\mathrm{P}$ (Mel); 50,66 $\mathrm{g} \mathrm{kg}^{-1}$ de matéria orgânica; $3,00 \mathrm{cmol}_{\mathrm{c}} \mathrm{dm}^{-3}$ de SB; 7,38 $\mathrm{cmol}_{\mathrm{c}} \mathrm{dm}^{-3}$ de CTC; $40,72 \%$ de saturação por bases; $460 \mathrm{~g} \mathrm{~kg}^{-1}$ de areia; $60 \mathrm{~g} \mathrm{~kg}^{-1}$ de silte e $480 \mathrm{~g} \mathrm{~kg}^{-1}$ de argila. Utilizou-se o delineamento experimental em blocos casualizados, 
com quatro repetições. As parcelas experimentais, com área de $9 \mathrm{~m}^{2}$, foram constituídas de seis linhas espaçadas de 0,50 m com $3 \mathrm{~m}$ de comprimento. $\mathrm{Na}$ área experimental, foi cultivada soja nos últimos três anos no sistema plantio direto. No período do experimento, a precipitação pluvial ( $\left.\mathrm{mm} \mathrm{mês}^{-1}\right)$ e a temperatura média mensal $\left({ }^{\circ} \mathrm{C}\right)$, respectivamente, nos meses de outubro, novembro e dezembro de 2004 e janeiro, fevereiro, março, abril e maio de 2005 , foram de: 162,5 e 24,$2 ; 199,8$ e 24,$3 ; 220,6$ e 23,$7 ; 181,4$ e 23,$7 ; 36,4$ e 24,$7 ; 201,6$ e 24,$1 ; 58,5$ e 24,6 ; e $21,7 \mathrm{~mm}$ e $22,0^{\circ} \mathrm{C}$.

A semeadura foi feita em 20 de outubro de 2004 , com a densidade de $12 \mathrm{~kg} \mathrm{ha}^{-1}$ de sementes de milheto, sendo as sementes colocadas em sulcos espaçados de $0,50 \mathrm{~m}$, previamente abertos e adubados com $250 \mathrm{~kg} \mathrm{ha}^{-1}$ da fórmula 8-16-16.

Os tratamentos foram constituídos pelas épocas de manejo do milheto: pré-emborrachamento (36 dias após a semeadura - DAS); pré-florescimento (45 DAS) e início do florescimento (52 DAS) e pelas épocas de coleta das bolsas de decomposição: 0, 30, 60, 90, 120 e 150 dias após sua instalação.

Em cada época de manejo, as plantas foram ceifadas e distribuídas uniformemente sobre o solo das parcelas. Retiraram-se, em cada parcela, amostras das plantas, para avaliação do teor de nutrientes e da matéria seca. Para avaliação da matéria seca, foram retiradas três amostras nas linhas centrais das parcelas, que foram secas em estufa à temperatura de $65{ }^{\circ} \mathrm{C}$, até atingirem peso constante. Para avaliar a taxa de decomposição da palhada, foi empregado o método das bolsas de decomposição (Thomas \& Asakawa, 1993), instaladas em dezembro de $2004 \mathrm{com}$ as dimensões de 0,15 x 0,20 m, confeccionadas de nylon, colocando cinco bolsas na superfície do solo, na linha central da parcela, contendo a matéria seca previamente triturada. Nessa avaliação, retirou-se uma bolsa de decomposição em cada época de avaliação. Após a coleta, o material foi retirado das bolsas e levado à estufa, com circulação e renovação forçada de ar, a $65^{\circ} \mathrm{C}$, por $72 \mathrm{~h}$, para secagem e posterior pesagem, para quantificação da matéria seca remanescente. Após a moagem das amostras, foram determinados, no material vegetal, os teores de $\mathrm{N}, \mathrm{P}, \mathrm{K}, \mathrm{Ca}, \mathrm{Mg}$ e S, segundo métodos descritos por Malavolta et al. (1997). $\mathrm{O} \mathrm{C}$ foi determinado pela queima em mufla à temperatura de $550{ }^{\circ} \mathrm{C}$ (Embrapa, 1997). As análises foram realizadas no material vegetal nos tempos: 0 (época de manejo), e nos materiais remanescentes nas bolsas de decomposição nos tempos 30, 60, 90, 120 e 150 dias.

Para descrever a liberação de nutrientes, nos diferentes estádios fenológicos da palhada remanescente, ajustou-se modelo matemático exponencial decrescente, descrito por Wieder \& Lang (1982) e utilizado por Thomas \& Asakawa (1993) e Boer et al. (2007): $\mathrm{Q}=\mathrm{Q}_{\mathrm{o}} \exp (-\mathrm{kt})$, em que: $\mathrm{Q}=$ quantidade de nutriente existente no tempo $t$, em dias (kg ha-1); $\mathrm{Q}_{\mathrm{o}}=$ fração de nutrientes potencialmente liberados $\left(\mathrm{kg} \mathrm{ha}^{-1}\right)$, e k = constante de liberação de nutrientes $\left(\mathrm{g} \mathrm{g}^{-1}\right)$. A partir dos valores da constante de liberação de nutrientes $(\mathrm{k})$, calculou-se o tempo de meia-vida $\left(\mathrm{T}_{1 / 2}\right)$, utilizando-se a fórmula $\mathrm{T}_{1 / 2}=0,693 /$ k (Paul \& Clark, 1989).

Os resultados obtidos foram submetidos à análise de variância e as médias comparadas pelo teste de Tukey a $5 \%$. As análises de regressão relativas à liberação de nutrientes até 150 dias após o manejo do milheto, nos diferentes estádios fenológicos, foram efetuadas com o uso do aplicativo SigmaPlot da Jandel Scientific e, para comparações entre as regressões, após linearização, foi utilizado o procedimento descrito em Snedecor \& Cochran (1989).

\section{RESULTADOS E DISCUSSÃO}

Analisando o acúmulo dos nutrientes nos diferentes estádios fenológicos do milheto, cultivar ADR300, observou-se que ocorreu aumento nas quantidades de nutrientes remanescentes com o avanço dos estádios fenológicos no momento do manejo (Quadro 1). Para avaliar o potencial de acúmulo de nutrientes da cultura do milheto, Oliveira et al. (2002), em estudo com milheto, sorgo, milho, mucuna-preta e feijão-de-porco, relataram o milheto como a cultura de maior acúmulo de macronutrientes a serem retornados ao solo para o próximo cultivo. Também Boer et al. (2007), em estudo com milheto, cultivar ADR500, no período de safrinha, manejado no pleno florescimento, encontraram, respectivamente, 416,94, 121,75 e $76,31 \mathrm{~kg} \mathrm{ha}{ }^{-1}$ para o $\mathrm{K}, \mathrm{N}$ e $\mathrm{Ca}$, em $10,8 \mathrm{Mg} \mathrm{ha}^{-1} \mathrm{de}$ matéria seca. Braz et al. (2004), na avaliação do milheto 52 dias após a germinação, obtiveram 348, 36,314 e $135 \mathrm{~kg} \mathrm{ha}^{-1}$, respectivamente, de N, P, K e $\mathrm{Ca}$. A partir destes resultados, pode-se observar a grande capacidade do milheto em acumular $\mathrm{K}$ na matéria seca. Já Oliveira et al. (2002), 100 dias após a semeadura, no estádio de florescimento, obtiveram para o $\mathrm{N}$ e P, respectivamente, 162,7 e 24,8 $\mathrm{kg} \mathrm{ha}^{-1} \mathrm{e}$ para o Ca e S, 93,3 e 25,6 kg ha-1, com a produção de $14,18 \mathrm{Mg} \mathrm{ha}^{-1}$ de matéria seca de milheto comum. Observou-se, também, pelos resultados experimentais obtidos, que a quantidade de nutrientes acumulados na matéria seca do milheto e o fornecimento para a cultura seguinte estão relacionados com o cultivar utilizado, com o estádio fenológico de manejo e com as condições climáticas.

Quanto ao Mg (Quadro 1), ocorreu acúmulo no início do florescimento, superior ao obtido por Teixeira et al. (2005), com 4,6 kg ha-1, e inferior ao encontrado por Boer et al. (2007), com o cultivar ADR500, e de Braz et al. (2004), com o cultivar BN2, com 39,76 e $52 \mathrm{~kg} \mathrm{ha}^{-1} \mathrm{de} \mathrm{Mg}$, respectivamente. 
Quadro 1. Nutrientes remanescentes na matéria seca do milheto ADR300 ceifado em diferentes estádios fenológicos, em bolsas de decomposição, até 150 dias após o manejo

\begin{tabular}{|c|c|c|c|c|c|c|}
\hline Estádio fenológico & $\mathbf{N}$ & $\mathbf{P}$ & $\mathbf{K}$ & $\mathbf{S}$ & $\mathbf{C a}$ & Mg \\
\hline & \multicolumn{6}{|c|}{0 dia após o corte ${ }^{(1)}$} \\
\hline Pré-emborrachamento & $88,05 \mathrm{~b}$ & $8,14 \mathrm{c}$ & $246,20 \mathrm{~b}$ & $0,38 \mathrm{c}$ & $23,12 \mathrm{c}$ & $10,78 \mathrm{c}$ \\
\hline Pré-florescimento & $100,80 \mathrm{~b}$ & $10,34 \mathrm{~b}$ & $370,90 a$ & $0,50 \mathrm{~b}$ & $32,14 \mathrm{~b}$ & $21,36 b$ \\
\hline \multirow[t]{2}{*}{ Início do florescimento } & $204,80 a$ & $25,28 \mathrm{a}$ & $447,60 \mathrm{a}$ & $1,25 \mathrm{a}$ & $58,79 a$ & $28,60 \mathrm{a}$ \\
\hline & \multicolumn{6}{|c|}{30 dias após o corte } \\
\hline Pré-emborrachamento & $20,19 \mathrm{c}$ & $2,83 \mathrm{~b}$ & $3,30 \mathrm{c}$ & $0,09 \mathrm{~b}$ & $7,91 \mathrm{c}$ & $2,72 \mathrm{c}$ \\
\hline Pré-florescimento & $36,56 \mathrm{~b}$ & $4,90 \mathrm{~b}$ & $6,58 \mathrm{~b}$ & $0,18 \mathrm{a}$ & $15,09 \mathrm{~b}$ & $5,39 \mathrm{~b}$ \\
\hline \multirow[t]{2}{*}{ Início do florescimento } & $73,88 \mathrm{a}$ & $10,07 a$ & $15,46 \mathrm{a}$ & $0,25 \mathrm{a}$ & $30,97 a$ & $12,77 \mathrm{a}$ \\
\hline & \multicolumn{6}{|c|}{60 dias após o corte } \\
\hline Pré-emborrachamento & $15,90 \mathrm{c}$ & $2,54 \mathrm{~b}$ & $2,76 b$ & $0,08 \mathrm{~b}$ & $7,89 \mathrm{c}$ & $2,62 \mathrm{c}$ \\
\hline Pré-florescimento & $30,29 b$ & $4,04 \mathrm{~b}$ & $4,20 \mathrm{~b}$ & $0,13 \mathrm{ab}$ & $11,05 \mathrm{~b}$ & $3,78 b$ \\
\hline \multirow[t]{2}{*}{ Início do florescimento } & $52,28 \mathrm{a}$ & $7,91 \mathrm{a}$ & $9,77 \mathrm{a}$ & $0,18 \mathrm{a}$ & $17,99 \mathrm{a}$ & $7,90 \mathrm{a}$ \\
\hline & \multicolumn{6}{|c|}{90 dias após o corte } \\
\hline Pré-emborrachamento & $12,87 \mathrm{~b}$ & $1,93 b$ & $1,32 \mathrm{c}$ & $0,07 \mathrm{~b}$ & $4,53 \mathrm{c}$ & $1,68 \mathrm{c}$ \\
\hline Pré-florescimento & $23,58 \mathrm{~b}$ & $3,67 \mathrm{~b}$ & $2,73 \mathrm{~b}$ & $0,13 \mathrm{ab}$ & $10,14 \mathrm{~b}$ & $3,61 b$ \\
\hline \multirow[t]{2}{*}{ Início do florescimento } & $48,91 \mathrm{a}$ & $7,26 \mathrm{a}$ & $8,76 \mathrm{a}$ & $0,18 \mathrm{a}$ & $17,02 \mathrm{a}$ & $7,00 \mathrm{a}$ \\
\hline & \multicolumn{6}{|c|}{120 dias após o corte } \\
\hline Pré-emborrachamento & $11,06 \mathrm{~b}$ & $1,79 b$ & $1,21 \mathrm{c}$ & $0,07 \mathrm{~b}$ & $4,27 \mathrm{~b}$ & $1,51 \mathrm{c}$ \\
\hline Pré-florescimento & $20,86 b$ & $2,35 \mathrm{~b}$ & $2,05 \mathrm{~b}$ & $0,12 \mathrm{ab}$ & $5,23 \mathrm{~b}$ & $2,15 b$ \\
\hline \multirow[t]{2}{*}{ Início do florescimento } & $35,17 \mathrm{a}$ & $6,17 \mathrm{a}$ & $7,09 a$ & $0,17 \mathrm{a}$ & $13,75 a$ & $5,60 \mathrm{a}$ \\
\hline & \multicolumn{6}{|c|}{150 dias após o corte } \\
\hline Pré-emborrachamento & $10,94 \mathrm{~b}$ & $1,64 b$ & $1,19 \mathrm{~b}$ & $0,07 \mathrm{a}$ & $4,26 \mathrm{~b}$ & $1,41 b$ \\
\hline Pré-florescimento & $16,70 \mathrm{~b}$ & $2,04 \mathrm{~b}$ & $1,62 b$ & $0,12 \mathrm{a}$ & $4,85 \mathrm{~b}$ & $1,79 b$ \\
\hline Início do florescimento & $33,13 a$ & $6,10 \mathrm{a}$ & $6,87 \mathrm{a}$ & $0,14 \mathrm{a}$ & $13,29 \mathrm{a}$ & $5,38 \mathrm{a}$ \\
\hline
\end{tabular}

Quanto à liberação de nutrientes da matéria seca do milheto, manejado no início do florescimento (Quadro 1), houve mineralização após 150 dias, de 84, 76, 98, 89, 77 e $81 \%$, respectivamente, de $\mathrm{N}, \mathrm{P}, \mathrm{K}, \mathrm{S}, \mathrm{Ca}$ e $\mathrm{Mg}$. Para Moraes (2001), em estudo com milheto comum, 168 dias após o manejo, obteve-se a liberação de $\mathrm{N}$, $\mathrm{P}, \mathrm{Ca}, \mathrm{Mg}$, e $\mathrm{S}$ de $96,0,93,2,97,6,87,6$ e 81,6 \%, respectivamente, superior à do presente trabalho.
Essa diferença de liberação de nutrientes está relacionada com as condições climáticas e com os cultivares utilizados.

A meia-vida do N, P, K, Ca, Mg e S (Quadro 2), calculada a partir dos modelos descritos para liberação dos nutrientes (Quadro 3), variou nos diferentes estádios fenológicos, sendo K o nutriente mais facilmente liberado, enquanto os mais resistentes à liberação foram o $\mathrm{P}$ e o $\mathrm{Ca}$. Boer et 
al. (2007), avaliando a liberação de nutrientes de milheto, cultivar ADR500, encontraram valores de meia-vida de 110 dias para N, 92 dias para $\mathrm{P}, 16$ dias para K, 74 dias para $\mathrm{Ca}, 107$ dias para $\mathrm{Mg}$ e 87 dias para S. A menor meia-vida para estes nutrientes obtida no presente estudo (Quadro 2) pode ser justificada pela diferença nos estádios fenológicos de manejo, pelo elevado índice pluviométrico e temperaturas elevadas (média de $24^{\circ} \mathrm{C}$ no período), na região do estudo, indicando maior decomposição da matéria seca remanescente e, conseqüentemente, maior liberação de nutrientes.

Quadro 2. Meia-vida dos nutrientes remanescentes na matéria seca do milheto ADR300 ceifado em diferentes estádios fenológicos

\begin{tabular}{llllllll}
\hline \multicolumn{1}{c}{ Estádio fenológico } & \multicolumn{6}{c}{ Meia - vida } \\
\hline & $\mathrm{N}$ & $\mathrm{P}$ & $\mathrm{K}$ & $\mathrm{Ca}$ & $\mathrm{Mg}$ & $\mathrm{S}$ \\
\cline { 2 - 7 } & \multicolumn{6}{c}{ dia } \\
Pré-emborrachamento & 20 & 41 & 5 & 38 & 24 & 24 \\
Pré-florescimento & 38 & 54 & 5 & 48 & 21 & 40 \\
Início do florescimento & 35 & 48 & 6 & 49 & 41 & 17 \\
\hline
\end{tabular}

O K teve a mineralização mais rápida, fato atestado pela análise do tempo de meia-vida (Quadro 2). Esta baixa meia-vida do $\mathrm{K}$ deve estar relacionada com o fato de o elemento não estar associado a componente estrutural do tecido vegetal e encontrar-se na forma iônica (Marschner, 1995; Bartz, 1998). Por esta razão, esse elemento pode ser facilmente extraído das plantas, tanto pela água da chuva, como pela própria umidade do solo.

A mineralização mais lenta foi a do $\mathrm{Ca}$, seguida do $\mathrm{P}$ e $\mathrm{Mg}$, no estádio do início do florescimento (Quadro 2). O Ca é um elemento que faz parte da composição estrutural das células (como parede celular), sendo co-fator de algumas enzimas envolvidas na hidrólise de ATP e de fosfolipídios e mensageiro secundário na regulação metabólica (Taiz \& Zeiger, 2004), tendo assim maior dificuldade de ser mineralizado e liberado para o solo.

Comparando as equações de regressão para a liberação de $\mathrm{K}$, estas não apresentaram diferenças significativas entre os diferentes estádios fenológicos, bem como para o S nos estádios de préemborrachamento vs pré-florescimento e também do pré-florescimento vs início do florescimento (Quadro 4).

Quadro 3. Coeficientes da equação de regressão, $Q$ = Qo exp (-kt), para N, P, K, S, Ca e Mg remanescentes até 150 dias após a dessecação

\begin{tabular}{|c|c|c|c|c|c|}
\hline \multirow{2}{*}{ Nutriente } & \multirow{2}{*}{ Coeficiente } & \multirow{2}{*}{ Unidade } & \multicolumn{3}{|c|}{ Estádio fenológico } \\
\hline & & & Pré-emborrachamento & Pré-florescimento & Início do floresciment \\
\hline \multirow{3}{*}{$\mathrm{N}$} & Qo & $\mathrm{kg} \mathrm{ha} \mathrm{h}^{-1}$ & 85,97 & 92,94 & 191,30 \\
\hline & $\mathrm{K}$ & $\mathrm{g} \mathrm{g}^{-1}$ & 0,0353 & 0,0118 & 0,0199 \\
\hline & $\mathrm{R}^{2}$ & & $0,91^{*}$ & $0,88^{*}$ & $0,89^{*}$ \\
\hline \multirow{3}{*}{$\mathrm{P}$} & Qo & $\mathrm{kg} \mathrm{ha-1}$ & 7,38 & 9,50 & 22,64 \\
\hline & $\mathrm{k}$ & $\mathrm{g} \mathrm{g}^{-1}$ & 0,0169 & 0,0128 & 0,0144 \\
\hline & $\mathrm{R}^{2}$ & & $0,84^{*}$ & $0,91^{* *}$ & $0,82^{*}$ \\
\hline \multirow{3}{*}{$\mathrm{K}$} & Qo & kg ha-1 & 246,15 & 370,90 & 447,61 \\
\hline & $\mathrm{k}$ & $\mathrm{g} \mathrm{g}^{-1}$ & 0,143 & 0,1336 & 0,1107 \\
\hline & $\mathrm{R}^{2}$ & & $1,0 * *$ & $1,0^{* *}$ & $1,0^{* *}$ \\
\hline \multirow{3}{*}{$\mathrm{S}$} & Qo & $\mathrm{kg} \mathrm{ha} \mathrm{h}^{-1}$ & 0,36 & 0,45 & 1,23 \\
\hline & $\mathrm{k}$ & $\mathrm{g} \mathrm{g}^{-1}$ & 0,0285 & 0,0173 & 0,0418 \\
\hline & $\mathrm{R}^{2}$ & & $0,82^{*}$ & $0,81^{*}$ & $0,91^{*}$ \\
\hline \multirow{3}{*}{$\mathrm{Ca}$} & Qo & $\mathrm{kg} \mathrm{ha-1}$ & 21,3191 & 30,2288 & 55,045 \\
\hline & $\mathrm{k}$ & $\mathrm{g} \mathrm{g}^{-1}$ & 0,0183 & 0,0155 & 0,0142 \\
\hline & $\mathrm{R}^{2}$ & & $0,87^{*}$ & $0,94^{* *}$ & $0,92^{* *}$ \\
\hline \multirow{3}{*}{$\mathrm{Mg}$} & Qo & $\mathrm{kg} \mathrm{ha}^{-1}$ & 10,321 & 20,8332 & 26,8243 \\
\hline & $\mathrm{k}$ & $\mathrm{g} \mathrm{g}^{-1}$ & 0,0284 & 0,0334 & 0,0171 \\
\hline & $\mathrm{R}^{2}$ & & $0,89^{*}$ & $0,93^{*}$ & $0,91^{* *}$ \\
\hline
\end{tabular}

$* * \mathrm{e}^{*}$ significativos a 1 e $5 \%$. 
Quadro 4. Comparação das equações de regressão (teste F), para N, P, K, Ca, Mg e S remanescentes até 150 dias após o manejo para o milheto ADR300 em diferentes estádios fenológicos

\begin{tabular}{|c|c|c|c|c|c|c|}
\hline \multirow{2}{*}{ Estádio fenológico } & \multicolumn{6}{|c|}{$\mathbf{F}$} \\
\hline & $\mathbf{N}$ & $\mathbf{P}$ & $\mathbf{K}$ & $\mathbf{C a}$ & Mg & $\mathbf{S}$ \\
\hline Pré-emborrachamento vs pré-florescimento & * & * & NS & * & * & NS \\
\hline Pré-emborrachamento vs início do florescimento & ** & ** & NS & $* *$ & $* *$ & * \\
\hline Pré-florescimento vs início do florescimento & $* *$ & $* *$ & NS & $* *$ & $* *$ & NS \\
\hline \multicolumn{7}{|c|}{ Recalculando o F para os modelos que não diferenciaram estatisticamente } \\
\hline \multicolumn{3}{|c|}{ Pré-emborrachamento+ pré-florescimento + início do florescimento } & NS & & & \\
\hline \multicolumn{6}{|c|}{ Pré-emborrachamento + pré-florescimento $v s$ pré-florescimento + início do florescimento } & * \\
\hline
\end{tabular}

$(* *)$ Significativo a $1 \%,(*)$ significativo a $5 \%$, (NS) não-significativo.

A análise comparativa dos modelos de liberação dos nutrientes nos diferentes estádios fenológicos indica que N, P, Ca e Mg apresentaram dinâmicas diferentes, independentemente do estádio fenológico (Quadro 4). A possível explicação se deve ao fato de que, em cada estádio fenológico, o material vegetal apresentou variação na proporção de folhas e caule e, possivelmente, na quantidade de componentes lignificados, proporcionando diferenças na decomposição da palhada e na liberação dos nutrientes. Este fato pode ser comprovado pela relação $\mathrm{C} / \mathrm{N}$ que variou entre os estádios fenológicos de pré-emborrachamento, préflorescimento e início do florescimento, respectivamente, com valores de 18, 19 e 22 .

Para o K, comprovada a não-significância entre as equações, uniram-se os valores dos dias após o manejo e a quantidade de $\mathrm{K}$ remanescente na palhada, ajustando uma nova equação para os estádios de préemborrachamento + pré-florescimento + início do florescimento $=354,89 \exp (-0,1235 \mathrm{t}) \mathrm{com} \mathrm{R}^{2}=0,94^{*}$ (Quadro 4).

Por tudo isto apresentado, a dinâmica de liberação de nutrientes pela palhada do milheto nos diferentes estádios fenológicos (Quadro 1 e 4), antecedendo a semeadura da cultura de verão, assume importância na tomada de decisão quanto à adubação a ser empregada, tendo em vista o conhecimento da quantidade e da intensidade de nutrientes liberados, após a dessecação, em cada estádio fenológico. Isto representa importante reserva nutricional disponibilizada para a cultura de verão, diminuindo possíveis perdas e quantidade de fertilizantes aplicados.

\section{CONCLUSÕES}

1. O maior acúmulo de nutrientes foi no estádio fenológico de início do florescimento.
2. A dinâmica da liberação de nutrientes foi diferente em todos os estádios fenológicos com exceção para o $\mathrm{K}$.

3. O K foi o nutriente mais facilmente liberado, independentemente do estádio fenológico.

\section{LITERATURA CITADA}

BARTZ, H. Dinâmica dos nutrientes e adubação em sistema de produção sob plantio direto. In: FRIES, M.R. Plantio direto em solos arenosos: alternativas de manejo para a sustentabilidade agropecuária. Santa Maria, UFSM/ Pallotti, 1998. p.52-63.

BOER, C.A.; ASSIS, R.L.; SILVA, G.P.; BRAZ, A.J.B.P.; BARROSO, A.L.L.; CARGNELUTTI FILHO, A. \& PIRES, F.R. Ciclagem de nutrientes por plantas de cobertura na entressafra em um solo de Cerrado. Pesq. Agropec. Bras., 42:1269-1276, 2007.

BRAZ, A.J.B.P.; SILVEIRA, P.M.; KLIEMANN, H.J. \& ZIMMERMANN, J.P. Acumulação de nutrientes em folhas de milheto e dos capins braquiária e mombaça. Pesq. Agropec. Trop., 34:83-87, 2004.

EMPRESA BRASILEIRA DE PESQUISA AGROPECUÁRIA EMBRAPA. Centro Nacional de Pesquisa de Solos. Manual de métodos de análise de solo. 2.ed. Rio de Janeiro, 1997. 212p.

MALAVOLTA, E; VITTI, G.C. \& OLIVEIRA, S.A. Avaliação do estado nutricional de plantas: Princípios e aplicações. 2.ed. Piracicaba, Potafos, 1997. 319p.

MARSCHNER, H. Mineral nutrition of higher plants. 2.ed. San Diego, Academic Press, 1995. 889p.

MORAES, R.N.S. Decomposição das palhadas de sorgo e milheto, mineralização de nutrientes e seus efeitos no solo e na cultura do milho em plantio direto. Lavras, Universidade Federal de Lavras, 2001. 90p. (Tese de Mestrado) 
OLIVEIRA,T.K.; CARVALHO, G.J. \& MORAES, R.N.S Plantas de cobertura e seus efeitos sobre o feijoeiro em plantio direto. Pesq. Agropec. Bras., 37:1079-1087, 2002.

PAUL, E.A. \& CLARK, F.E. Soil microbiology and biochemistry. San Diego, Academic Press, 1989. 275p.

SNEDECOR, G.W. \& COCHRAN, W.G. Statistical methods. 8.ed. Ames, Iowa State University Press, 1989. 503p.

TAIZ, L. \& ZEIGER, E. Fisiologia vegetal. 3.ed. Porto Alegre, Artmed, 2004. 719p.
TEIXEIRA, C.M.; CARVALHO, G.J.C.; FURTINI NETO, A.E.; ANDRADE, M.J.B. \& MARQUES, E.L.S. Produção de biomassa e teor de macronutrientes do milheto, feijãode-porco e guandu-anão em cultivo solteiro e consorciado. Ci. Agrotec., 29:93-99, 2005.

THOMAS, R.J. \& ASAKAWA, N.M. Decomposition of leaf litter from tropical forage grasses and legumes. Soil Biol. Biochem., 23:1351-1361, 1993.

WIEDER, R.K. \& LANG, G.E. A critique of the analytical methods used in examining decomposition data obtained from litter bags. Ecology, 63:1636-1642, 1982. 
